\title{
On the magnitude of asymptotic probability measures of Dedekind zeta-functions and other Euler products
}

\author{
by
}

\author{
Kohji Matsumoto (Morioka)
}

1. Introduction. Let $s=\sigma+i t$ be a complex variable, $\zeta(s)$ the Riemann zeta-function, and let $R$ be any closed rectangle in the complex plane $\mathbf{C}$ with edges parallel to the axes. For any $\sigma>1 / 2$ and any $T>0$, we denote by $V(T, R, \sigma ; \zeta)$ the 1-dimensional Lebesgue measure of the set

$$
\{t \in[-T, T] \mid \log \zeta(\sigma+i t) \in R\} .
$$

Then the existence of the limit

$$
W(R, \sigma ; \zeta)=\lim _{T \rightarrow \infty} V(T, R, \sigma ; \zeta) / 2 T
$$

has been shown in Bohr-Jessen's classical work [1]. We can extend $W$ to a probability measure defined on $\mathbf{C}$, which we call the asymptotic probability measure of $\log \zeta(s)$.

Let $r>0$, and put

$$
R(r)=\{z \mid-r \leq \operatorname{Re} z \leq r,-r \leq \operatorname{Im} z \leq r\} .
$$

It is an interesting problem to study the behaviour of $W(R(r), \sigma ; \zeta)$ as $r$ tends to infinity. This problem is trivial if $\sigma>1$, because in this case $W(R(r), \sigma ; \zeta)=1$ for sufficiently large $r$. In case $1 / 2<\sigma \leq 1$, there is a result of Jessen and Wintner [4], which asserts that for any positive $r$ and $\lambda$, we have

$$
W(\mathbf{C}-R(r), \sigma ; \zeta) \leq C e^{-\lambda r^{2}},
$$

with a positive constant $C=C(\lambda, \sigma)$.

For a long time, (1.1) had been the only non-trivial result on the magnitude of $W$. Recently, Joyner [5, Theorem 4.3] proved the following nice improvement of (1.1): If $1 / 2<\sigma<1$, then

$$
\begin{aligned}
\exp \left(-C^{\prime} r^{1 /(1-\sigma)}(\log r)^{\sigma /(1-\sigma)}\right) & \leq W(\mathbf{C}-R(r), \sigma ; \zeta) \\
& \leq \exp \left(-C^{\prime \prime} r^{1 /(1-\sigma)}(\log r)^{\sigma /(1-\sigma)}\right)
\end{aligned}
$$


for any sufficiently large $r$, with positive constants $C^{\prime}$ and $C^{\prime \prime}$ which depend only on $\sigma$ and satisfy $C^{\prime}>C^{\prime \prime}$. (We should also mention here the paper of Nikishin [11] and extensive studies of A. Laurinchikas.)

On the other hand, the author [7] generalized Bohr-Jessen's existence theorem to the case of fairly general Euler products. The purpose of this paper is to discuss estimates of Joyner's type in such general situations, especially in the case of Dedekind zeta-functions.

Let $\mathbf{Q}$ be the rational number field, $F$ an algebraic number field of finite degree, $d=[F: \mathbf{Q}]$, and $d_{1}=\max \{d, 2\}$. We denote by $V\left(T, R, \sigma ; \zeta_{F}\right)$ the 1-dimensional Lebesgue measure of the set

$$
\left\{t \in[-T, T] \mid \log \zeta_{F}(\sigma+i t) \in R\right\},
$$

where $\zeta_{F}$ is the Dedekind zeta-function attached to $F$. (This definition depends on the choice of the branch of $\log \zeta_{F}(s)$. The rigorous definition is given in (2.3).) If $\sigma>1-d_{1}^{-1}$, then the limit

$$
W\left(R, \sigma ; \zeta_{F}\right)=\lim _{T \rightarrow \infty} V\left(T, R, \sigma ; \zeta_{F}\right) / 2 T
$$

exists. Concerning the magnitude of this asymptotic probability measure, we will prove the following

Corollary 1. Let $F$ be an arbitrary finite extension of $\mathbf{Q}, F^{*}$ the Galois closure of $F$, and $d^{*}=\left[F^{*}: \mathbf{Q}\right]$. Then

$$
\begin{aligned}
2^{-39} \exp \{ & \left.-M_{1}\left(d^{*}\right)^{2 \sigma}\left(d^{*} / d\right)^{1 /(1-\sigma)} r^{1 /(1-\sigma)}(\log r)^{\sigma /(1-\sigma)}(1+o(1))\right\} \\
& \leq W\left(\mathbf{C}-R(r), \sigma ; \zeta_{F}\right) \\
& \leq 4 \exp \left\{-M_{2}\left(d^{*}\right)^{-2} d^{-1 /(1-\sigma)} r^{1 /(1-\sigma)}(\log r)^{\sigma /(1-\sigma)}(1+o(1))\right\}
\end{aligned}
$$

(for $\left.1-d_{1}^{-1}<\sigma<1\right)$ and

$$
\begin{aligned}
2^{-39} \exp \{-100 & \left.\left(d^{*}\right)^{2} \exp \exp \left(2 d^{*} d^{-1} r(1+o(1))\right)\right\} \\
& \leq W\left(\mathbf{C}-R(r), 1 ; \zeta_{F}\right) \\
& \leq 4 \exp \left\{-(3 / 4)\left(d^{*}\right)^{-2} \exp \exp \left((2 d)^{-1} r(1+o(1))\right)\right\}
\end{aligned}
$$

for any large $r$, where $M_{1}$ and $M_{2}$ are positive constants which depend only on $\sigma$.

Corollary 2. In case $F$ is a Galois extension of $\mathbf{Q}$, we have

$$
\begin{aligned}
2^{-39} \exp \left\{-M_{1} d^{-1}\right. & \left.r^{1 /(1-\sigma)}(\log r)^{\sigma /(1-\sigma)}(1+o(1))\right\} \\
& \leq W\left(\mathbf{C}-R(r), \sigma ; \zeta_{F}\right) \\
& \leq 4 \exp \left\{-M_{2} d^{-1} r^{1 /(1-\sigma)}(\log r)^{\sigma /(1-\sigma)}(1+o(1))\right\}
\end{aligned}
$$


$\left(\right.$ for $\left.1-d_{1}^{-1}<\sigma<1\right)$ and

$$
\begin{aligned}
2^{-39} \exp \left\{-100 d^{-1}\right. & \exp \exp (2 r(1+o(1)))\} \\
& \leq W\left(\mathbf{C}-R(r), 1 ; \zeta_{F}\right) \\
& \leq 4 \exp \left\{-(3 / 4) d^{-1} \exp \exp \left(2^{-1} r(1+o(1))\right)\right\}
\end{aligned}
$$

for any large $r$.

Remark. We can write down explicit values of the constants $M_{1}$ and $M_{2}$. In fact, in our proofs it is shown that we can choose

$$
M_{1}=100(1-\sigma)^{-1}(2 \sigma-1) 2^{1 /(1-\sigma)}
$$

and

$$
M_{2}=(3 / 4)(1-\sigma)^{-1}(2 \sigma-1) 2^{-1 /(1-\sigma)} .
$$

These results are obtained as special cases of a general theorem, which will be stated in Section 4. We note that if $F=\mathbf{Q}$, then Corollary 2 implies Joyner's estimate (1.2). The proof of these corollaries will be given in Sections 5 and 6, and in Section 7 we will mention briefly other examples, such as zeta-functions attached to certain cusp forms.

In the following sections, $\mu_{N}$ denotes the $N$-dimensional Lebesgue measure. $\mathbf{T}^{N}$ is the $N$-dimensional unit torus $[0,1)^{N}$. The symbol \#S signifies the cardinality of the set $S$.

The author expresses his sincere gratitude to Professor Tetsuya Hattori for useful advice and discussions, especially concerning the proof of (4.12). $\mathrm{He}$ is also indebted to the referee for valuable comments.

2. Definitions. Let $\mathbf{N}$ be the set of positive integers, and $g$ a mapping from $\mathbf{N}$ to $\mathbf{N}$. For any $n \in \mathbf{N}$ and any $j$ with $1 \leq j \leq g(n)$, let $a_{n}^{(j)}$ be complex numbers and $f(j, n)$ positive integers. We define polynomials

$$
A_{n}(X)=\prod_{j=1}^{g(n)}\left(1-a_{n}^{(j)} X^{f(j, n)}\right),
$$

and put

$$
\varphi(s)=\prod_{n=1}^{\infty} A_{n}\left(p_{n}^{-s}\right)^{-1},
$$

where $p_{n}$ denotes the $n$th prime number. We assume the conditions

$$
|g(n)| \leq C_{1} p_{n}^{\alpha}, \quad\left|a_{n}^{(j)}\right| \leq p_{n}^{\beta},
$$

where $C_{1}$ is a positive constant, and $\alpha$ and $\beta$ are non-negative constants. Then the infinite product (2.1) converges absolutely in the region $\sigma>\alpha+$ $\beta+1$, and defines a holomorphic function $\varphi(s)$. Let $\varrho \geq \alpha+\beta+1 / 2$, and 
we assume that $\varphi(s)$ can be meromorphically continued to the half-plane $\sigma \geq \varrho$. Moreover, we make the following three assumptions:

(1) All poles of $\varphi(s)$ in this region are included in a compact set.

(2) $|\varphi(\sigma+i t)|=O\left(|t|^{C_{2}}\right)$ in this region with some constant $C_{2}$.

(3) There is no pole on the line $\sigma=\varrho$, and we have the mean-value estimate

$$
\int_{-T}^{T}|\varphi(\varrho+i t)|^{2} d t=O(T) .
$$

Let $\mathcal{S}$ be the set of all zeros and poles of $\varphi(s)$ in the region $\sigma \geq \varrho$, and we put

$$
G=\{s \mid \sigma \geq \varrho\}-\bigcup_{\sigma^{\prime}+i t^{\prime} \in \mathcal{S}}\left\{s=\sigma+i t^{\prime} \mid \varrho \leq \sigma \leq \sigma^{\prime}\right\} .
$$

For any $s_{0}=\sigma_{0}+i t_{0} \in G$, we define the value of $\log \varphi\left(s_{0}\right)$ by analytic continuation along the path $\left\{s=\sigma+i t_{0} \mid \sigma \geq \sigma_{0}\right\}$. Let $R$ be any closed rectangle with edges parallel to the axes, and

$$
V(T, R, \sigma ; \varphi)=\mu_{1}(\{t \in[-T, T] \mid \sigma+i t \in G, \log \varphi(\sigma+i t) \in R\}) .
$$

In [7] it is shown that for any $\sigma>\varrho$, the limit

$$
W(R, \sigma ; \varphi)=\lim _{T \rightarrow \infty} V(T, R, \sigma ; \varphi) / 2 T
$$

exists, and $W$ can be extended to a regular probability measure on $\mathbf{C}$.

Here we comment on the assumptions (1)-(3). Assumptions (1) and (2) are usually satisfied in practical applications. The assumption (3) is essential; in the case of $\zeta(s),(3)$ is valid only for $\varrho>1 / 2$, so Bohr and Jessen could prove their existence theorem only in the half-plane $\sigma>1 / 2$. In fact, A. Selberg's unpublished result shows that the situation of the valuedistribution is rather different on the line $\sigma=1 / 2$. See Joyner's book [5].

If $\varphi(s)$ has a functional equation, we can check the assumption (3) by the following general result.

Lemma 1 (Theorem 3 of Potter [12]). Let $l \geq 0$ and let $\gamma, \delta$ be real numbers. Suppose that the functions

$$
f_{1}(s)=\sum_{n=1}^{\infty} a_{n} l_{n}^{-s}, \quad f_{2}(s)=\sum_{n=1}^{\infty} b_{n} k_{n}^{-s}
$$

satisfy the following conditions:

(i) $\left\{l_{n}\right\}$ and $\left\{k_{n}\right\}$ are increasing sequences of positive numbers, tend to infinity as $n$ tends to infinity, and for any $\varepsilon>0$, there exists a positive integer $n_{0}(\varepsilon)$ for which

$$
\log \left(l_{n} / l_{n-1}\right)>l_{n}^{-(l+\varepsilon)}, \quad \log \left(k_{n} / k_{n-1}\right)>k_{n}^{-(l+\varepsilon)}
$$


for any $n \geq n_{0}(\varepsilon)$.

(ii) $a_{n}$ and $b_{n}$ are complex numbers satisfying the estimates

$$
\sum_{l_{n} \leq x}\left|a_{n}\right|^{2}=O\left(x^{\gamma+\varepsilon}\right), \quad \sum_{k_{n} \leq x}\left|b_{n}\right|^{2}=O\left(x^{\gamma+\varepsilon}\right)
$$

for any $x>0$ and any $\varepsilon>0$.

(iii) The functions $f_{1}(s)$ and $f_{2}(s)$ can be continued meromorphically to the whole plane, and the functional equation $f_{1}(s)=H(s) f_{2}(\delta-s)$ holds, where the factor $H(s)$ satisfies the estimates

$$
H(s)=O\left(|t|^{c((1 / 2) \delta-\sigma)}\right), \quad H(s)^{-1}=O\left(|t|^{c(\sigma-(1 / 2) \delta)}\right)
$$

with some $c>0$.

Then

$$
\lim _{T \rightarrow \infty}(2 T)^{-1} \int_{-T}^{T}\left|f_{1}(\sigma+i t)\right|^{2} d t=\sum_{n=1}^{\infty}\left|a_{n}\right|^{2} l_{n}^{-2 \sigma}
$$

for $\sigma>\max \left\{\delta / 2,(1 / 2)(\gamma+l)-c^{-1}\right\}$, and a similar result holds for $f_{2}(s)$.

The analogue of Jessen-Wintner's estimate (1.1) can be proved in this general setting. If $\sigma>\varrho$, then for any positive $r$ and $\lambda$,

$$
W(\mathbf{C}-R(r), \sigma ; \varphi) \leq C e^{-\lambda r^{2}}
$$

with a positive constant $C=C(\lambda, \sigma, \varphi)$. We will give a proof of this inequality in the last section.

As an example, consider the Dedekind zeta-function $\zeta_{F}(s)$. Let $\left\{\mathfrak{p}_{n}^{(1)}, \ldots, \mathfrak{p}_{n}^{(g(n))}\right\}$ be the set of all prime divisors of $p_{n}$ in $F$. We define the integers $e(j, n)$ and $f(j, n)(1 \leq j \leq g(n))$ by

$$
p_{n}=\prod_{j=1}^{g(n)}\left(\mathfrak{p}_{n}^{(j)}\right)^{e(j, n)} \quad \text { and } \quad N \mathfrak{p}_{n}^{(j)}=p_{n}^{f(j, n)},
$$

where $N \mathfrak{p}_{n}^{(j)}$ denotes the norm of $\mathfrak{p}_{n}^{(j)}$. Then we can write

$$
\zeta_{F}(s)=\prod_{n=1}^{\infty} \prod_{j=1}^{g(n)}\left(1-p_{n}^{-f(j, n) s}\right)^{-1},
$$

which is of the form $(2.1)$ with $a_{n}^{(j)}=1$ for any $n$ and $j$. In case $F$ is a Galois extension of $\mathbf{Q}$, the value of $e(j, n)$ (resp. $f(j, n)$ ) is independent of $j$; denote it by $e(n)$ (resp. $f(n))$. The values of $e(n), f(n)$ and $g(n)$ of course depend on $F$. For an arbitrary $F$, we denote by $e^{*}(n), f^{*}(n)$ and $g^{*}(n)$ the corresponding values for the Galois closure $F^{*}$. Later, we sometimes use the notations $g(p)=g(n)$ and $g^{*}(p)=g^{*}(n)$ for $p=p_{n}$. 
We fix a real number $\sigma>1-d_{1}^{-1}$, and we choose a $\varrho$ which satisfies $1-d_{1}^{-1}<\varrho<\sigma$. By using Lemma 1 , or a result of Chandrasekharan and Narasimhan [2], we see the assumption (3) is satisfied for this $\varrho$. Hence, the limit (2.4) exists for any $\sigma>\varrho$, in particular for our fixed $\sigma$. This completes the proof of (1.3). (See also [8], where an alternative method is developed.)

3. The mapping $S_{N}$. Let $N \in \mathbf{N}$, and define

$$
\varphi_{N}(s)=\prod_{n=1}^{N} A_{n}\left(p_{n}^{-s}\right)^{-1}
$$

and

$$
V_{N}(T, R, \sigma ; \varphi)=\mu_{1}\left(\left\{t \in[-T, T] \mid \log \varphi_{N}(\sigma+i t) \in R\right\}\right) .
$$

Also we define the mapping $S_{N}$ from $\mathbf{T}^{N}$ to $\mathrm{C}$ by

$$
S_{N}\left(\theta_{1}, \ldots, \theta_{N}\right)=-\sum_{n=1}^{N} \sum_{j=1}^{g(n)} \log \left(1-a_{n}^{(j)} p_{n}^{-f(j, n) \sigma} \exp \left(2 \pi i f(j, n) \theta_{n}\right)\right),
$$

where $\left(\theta_{1}, \ldots, \theta_{N}\right) \in[0,1)^{N}=\mathbf{T}^{N}$, and the logarithmic function on the right-hand side takes the principal value. In [7], we have shown that the limit

exists and

$$
W_{N}(R, \sigma ; \varphi)=\lim _{T \rightarrow \infty} V_{N}(T, R, \sigma ; \varphi) / 2 T
$$

$$
W_{N}(R, \sigma ; \varphi)=\mu_{N}\left(S_{N}^{-1}(R)\right) .
$$

We can extend $W_{N}$ to a regular probability measure on $\mathbf{C}$ by defining

$$
W_{N}(E, \sigma ; \varphi)=\mu_{N}\left(S_{N}^{-1}(E)\right)
$$

for any Borel set $E \subset \mathbf{C}$. In [7], we have shown that there is a subsequence of $\left\{W_{N}\right\}_{N=1}^{\infty}$ which converges weakly to a regular probability measure $W=$ $W(\cdot, \sigma ; \varphi)$, and for any rectangle $R$

$$
W(R, \sigma ; \varphi)=\lim _{N \rightarrow \infty} W_{N}(R, \sigma ; \varphi),
$$

which coincides with the limit (2.4).

Now we assume $\sigma>\alpha+\beta+1 / 2$. We write $\theta=\left(\theta_{1}, \ldots, \theta_{N}\right)$ and $S_{N}(\theta)=S_{N}\left(\theta_{1}, \ldots, \theta_{N}\right)$. Then

$$
\begin{aligned}
S_{N}(\theta) & =-\sum_{n=1}^{N} \sum_{j=1}^{g(n)} \log \left\{1-a_{n}^{(j)} p_{n}^{-f(j, n) \sigma} \exp \left(2 \pi i f(j, n) \theta_{n}\right)\right\} \\
& =\sum_{n=1}^{N} \sum_{j=1}^{g(n)} \sum_{m=1}^{\infty} m^{-1}\left(a_{n}^{(j)} p_{n}^{-f(j, n) \sigma}\right)^{m} \exp \left(2 \pi i m f(j, n) \theta_{n}\right)
\end{aligned}
$$




$$
\begin{aligned}
= & \sum_{n=1}^{N} \sum_{\substack{1 \leq j \leq g(n) \\
f(j, n)=1}} a_{n}^{(j)} p_{n}^{-\sigma} \exp \left(2 \pi i \theta_{n}\right) \\
& +\sum_{n=1}^{N} \sum_{\substack{1 \leq j \leq g(n) \\
f(j, n) \geq 2}} a_{n}^{(j)} p_{n}^{-f(j, n) \sigma} \exp \left(2 \pi i f(j, n) \theta_{n}\right) \\
& +\sum_{n=1}^{N} \sum_{j=1}^{g(n)} \sum_{m \geq 2} m^{-1}\left(a_{n}^{(j)} p_{n}^{-f(j, n) \sigma}\right)^{m} \exp \left(2 \pi i m f(j, n) \theta_{n}\right) \\
= & S_{N}^{*}(\theta)+S_{N}^{(1)}(\theta)+S_{N}^{(2)}(\theta), \text { say. }
\end{aligned}
$$

By using (2.2), we see that

$$
\left|S_{N}^{(2)}(\theta)\right| \leq C_{1} \sum_{m \geq 2} m^{-1} \sum_{n=1}^{\infty} p_{n}^{\alpha-m(\sigma-\beta)}
$$

and

so

$$
\begin{aligned}
\sum_{n=1}^{\infty} p_{n}^{\alpha-m(\sigma-\beta)} & \leq 2^{\alpha-m(\sigma-\beta)}+\int_{2}^{\infty} x^{\alpha-m(\sigma-\beta)} d x \\
& \leq\left(1+\frac{2}{2(\sigma-\beta)-1-\alpha}\right) 2^{\alpha-m(\sigma-\beta)}
\end{aligned}
$$

$$
\left|S_{N}^{(2)}(\theta)\right| \leq A_{1}
$$

with a positive constant $A_{1}$ depending only on $\sigma$ and $\varphi$. Also, we see that

$$
\left|S_{N}^{(1)}(\theta)\right| \leq C_{1} \sum_{n=1}^{\infty} p_{n}^{\alpha+\beta-2 \sigma}=A_{2}, \text { say. }
$$

Hence,

$$
\left|S_{N}(\theta)-S_{N}^{*}(\theta)\right| \leq A,
$$

where the positive constant $A=A_{1}+A_{2}$ depends only on $\sigma$ and $\varphi$.

Next, let

$$
B_{n}=\sum_{\substack{1 \leq j \leq g(n) \\ f(j, n)=1}} a_{n}^{(j)} p_{n}^{-\sigma},
$$

and $u_{n}=\theta_{n}+\arg \left(B_{n}\right)$. Then we have

$$
S_{N}^{*}(\theta)=\sum_{n=1}^{N} B_{n} \exp \left(2 \pi i \theta_{n}\right)=\sum_{n=1}^{N}\left|B_{n}\right| \exp \left(2 \pi i u_{n}\right)
$$




$$
\begin{aligned}
& =\sum_{n=1}^{N}\left|B_{n}\right| \cos \left(2 \pi u_{n}\right)+i \sum_{n=1}^{N}\left|B_{n}\right| \sin \left(2 \pi u_{n}\right) \\
& =X_{N}(u)+i Y_{N}(u), \text { say, }
\end{aligned}
$$

where we write $u=\left(u_{1}, \ldots, u_{N}\right)$. Let $P_{N}$ (resp. $\left.Q_{N}\right)$ be the probability measure on the real number field $\mathbf{R}$, defined as

$$
P_{N}(E)=\mu_{N}\left(X_{N}^{-1}(E)\right) \quad\left(\text { resp. } Q_{N}(E)=\mu_{N}\left(Y_{N}^{-1}(E)\right)\right)
$$

for any Borel subset $E \subset \mathbf{R}$. Let $r$ be an arbitrary positive number greater than $A$. From (3.1) we see

$$
\left\{\theta \mid S_{N}^{*}(\theta) \in R(r-A)\right\} \subset\left\{\theta \mid S_{N}(\theta) \in R(r)\right\} .
$$

Also, it is clear that

$$
\mathbf{C}-R(r-A) \subset\{z \in \mathbf{C}|| \operatorname{Re} z \mid>r-A\} \cup\{z \in \mathbf{C}|| \operatorname{Im} z \mid>r-A\} .
$$

Hence we have

$$
\begin{aligned}
& W_{N}(\mathbf{C}-R(r), \sigma ; \varphi) \\
& \quad=\mu_{N}\left(\left\{\theta \mid S_{N}(\theta) \in \mathbf{C}-R(r)\right\}\right) \\
& \quad \leq \mu_{N}\left(\left\{\theta \mid S_{N}^{*}(\theta) \in \mathbf{C}-R(r-A)\right\}\right) \\
& \quad \leq \mu_{N}\left(\left\{u|| X_{N}(u) \mid>r-A\right\}\right)+\mu_{N}\left(\left\{u|| Y_{N}(u) \mid>r-A\right\}\right) \\
& \quad=P_{N}(I(r-A))+Q_{N}(I(r-A)),
\end{aligned}
$$

where we use the notation $I(a)=(-\infty,-a) \cup(a, \infty)$. Similarly, by using

$$
\left\{\theta \mid S_{N}(\theta) \in R(r)\right\} \subset\left\{\theta \mid S_{N}^{*}(\theta) \in R(r+A)\right\}
$$

and

we obtain

$$
\{z \in \mathbf{C}|| \operatorname{Re} z \mid>r+A\} \subset \mathbf{C}-R(r+A),
$$

$$
W_{N}(\mathbf{C}-R(r), \sigma ; \varphi) \geq P_{N}(I(r+A)) .
$$

4. Application of Montgomery's lemma. Let $\mathbf{T}^{\infty}$ be the infinite direct product of $\mathbf{T}^{1}, \bar{u}=\left(u_{n}\right) \in \mathbf{T}^{\infty}$, and define the mapping $x_{n}$ (resp. $y_{n}$ ) from $\mathbf{T}^{\infty}$ to $\mathbf{R}$ by

$$
x_{n}(\bar{u})=\left|B_{n}\right| \cos \left(2 \pi u_{n}\right) \quad\left(\text { resp. } y_{n}(\bar{u})=\left|B_{n}\right| \sin \left(2 \pi u_{n}\right)\right) .
$$

We consider $\mathbf{T}^{\infty}$ as a probability space with the probability measure $\mu_{\infty}$ defined as the infinite direct product of $\mu_{1}$. Then $x_{n}$ is a real-valued random variable, and it is easily seen that the $x_{n}$ 's are independent. We define

$$
X(\bar{u})=\sum_{n=1}^{\infty} x_{n}(\bar{u})=\sum_{n=1}^{\infty}\left|B_{n}\right| \cos \left(2 \pi u_{n}\right) .
$$


The expectation and the variance of $x_{n}$ are 0 and $\left|B_{n}\right|^{2} / 2$, respectively. Since

$$
\sum_{n=1}^{\infty}\left|B_{n}\right|^{2}<\infty
$$

in the region $\sigma>\alpha+\beta+1 / 2$, by Kolmogorov's theorem, (4.1) is convergent almost everywhere. Similarly,

$$
Y(\bar{u})=\sum_{n=1}^{\infty} y_{n}(\bar{u})=\sum_{n=1}^{\infty}\left|B_{n}\right| \sin \left(2 \pi u_{n}\right)
$$

is convergent almost everywhere. By $P$ (resp. $Q$ ) we denote the probability measure defined as

$$
P(E)=\mu_{\infty}\left(X^{-1}(E)\right) \quad\left(\text { resp. } Q(E)=\mu_{\infty}\left(Y^{-1}(E)\right)\right)
$$

for any Borel set $E \subset \mathbf{R}$.

Now we quote the following

LEMMA 2 (Montgomery [9]). Let $\left\{r_{k}\right\}$ be any sequence of non-negative numbers, positive for infinitely many $k$ 's, satisfying the conditions

(i) $\lim _{k \rightarrow \infty} r_{k}=0$,

(ii) $\sum_{k=1}^{\infty} r_{k}^{2}<\infty$.

Let $\theta_{k} \in \mathbf{T}^{1}, \bar{\theta}=\left(\theta_{k}\right) \in \mathbf{T}^{\infty}$, and define

$$
f(\bar{\theta})=\sum_{k=1}^{\infty} r_{k} \cos \left(2 \pi \theta_{k}\right), \quad g(\bar{\theta})=\sum_{k=1}^{\infty} r_{k} \sin \left(2 \pi \theta_{k}\right) .
$$

(By Kolmogorov's theorem, these are convergent almost everywhere.) Then, for any $K \in \mathbf{N}$,

$$
\mu_{\infty}\left(f(\bar{\theta})>2 \sum_{k=1}^{K} r_{k}\right) \leq \exp \left\{-(3 / 4)\left(\sum_{k=1}^{K} r_{k}\right)^{2}\left(\sum_{k>K} r_{k}^{2}\right)^{-1}\right\},
$$

and the same estimate holds for $g(\bar{\theta})$. Moreover, if we assume the additional condition

(iii) $r_{k}$ decreases monotonically,

then

$$
\mu_{\infty}\left(f(\bar{\theta})>2^{-1} \sum_{k=1}^{K} r_{k}\right) \geq 2^{-40} \exp \left\{-100\left(\sum_{k=1}^{K} r_{k}\right)^{2}\left(\sum_{k>K} r_{k}^{2}\right)^{-1}\right\},
$$

and the same estimate holds for $g(\bar{\theta})$.

In Montgomery's statement, (iii) is also assumed in the upper-bound estimate. However, (iii) is required only for the lower-bound part of the 
proof presented in [9]. (It is, as Montgomery said, not the original proof of Montgomery himself, but a simplified one due to A. M. Odlyzko. See also Montgomery-Odlyzko [10].)

We apply this lemma to the case $r_{k}=\left|B_{k}\right|, \theta_{k}=u_{k}, f(\bar{\theta})=X(\bar{u})$ and $g(\bar{\theta})=Y(\bar{u})$. The condition (i) can be easily checked, and (ii) is (4.2). Hence, for any $K \in \mathbf{N}$,

$$
\mu_{\infty}\left(X(\bar{u})>2 \sum_{k=1}^{K}\left|B_{k}\right|\right) \leq \exp \left\{-(3 / 4)\left(\sum_{k=1}^{K}\left|B_{k}\right|\right)^{2}\left(\sum_{k>K}\left|B_{k}\right|^{2}\right)^{-1}\right\} .
$$

If $\bar{u}=\left(u_{k}\right)$ satisfies $X(\bar{u}) \geq a$ (where $a$ is an arbitrary positive number), then $\bar{u}+1 / 2=\left(u_{k}+1 / 2\right)$ satisfies $X(\bar{u}+1 / 2) \leq-a$, and vice versa. Hence,

$$
\begin{aligned}
\mu_{\infty}\left(|X(\bar{u})|>2 \sum_{k=1}^{K}\right. & \left.\left|B_{k}\right|\right) \\
& \leq 2 \exp \left\{-(3 / 4)\left(\sum_{k=1}^{K}\left|B_{k}\right|\right)^{2}\left(\sum_{k>K}\left|B_{k}\right|^{2}\right)^{-1}\right\} .
\end{aligned}
$$

For any given $r$, we choose $K=K(r)$ which satisfies the condition

$$
2 \sum_{k=1}^{K}\left|B_{k}\right|<r-A \leq 2 \sum_{k=1}^{K+1}\left|B_{k}\right|
$$

and put

$$
\eta=r-A-2 \sum_{k=1}^{K}\left|B_{k}\right|
$$

Then (4.3) implies

$$
P(I(r-A-\eta)) \leq 2 \exp \left\{-(3 / 4)\left(\sum_{k=1}^{K}\left|B_{k}\right|\right)^{2}\left(\sum_{k>K}\left|B_{k}\right|^{2}\right)^{-1}\right\},
$$

and the same estimate holds for $Q(I(r-A-\eta))$.

Let $\xi(t)$ be a continuous function which equals 1 if $t \in[-(r-A-$ $\left.\left.2^{-1} \eta\right), r-A-2^{-1} \eta\right]$, equals 0 if $t \in I(r-A)$, and satisfies $0 \leq \xi(t) \leq 1$ for any real $t$. Then, from (3.3), we have

$$
\begin{aligned}
W_{N}(\mathbf{C}-R(r), \sigma ; \varphi) & \leq 2-P_{N}([-(r-A), r-A])-Q_{N}([-(r-A), r-A]) \\
& \leq 2-\int_{-\infty}^{\infty} \xi(t) d P_{N}(t)-\int_{-\infty}^{\infty} \xi(t) d Q_{N}(t)
\end{aligned}
$$


for any $N$. As $N$ tends to infinity, it follows that

$$
\begin{aligned}
& W(\mathbf{C}-R(r), \sigma ; \varphi) \\
& \quad \leq 2-\int_{-\infty}^{\infty} \xi(t) d P(t)-\int_{-\infty}^{\infty} \xi(t) d Q(t) \\
& \quad \leq 2-P([-(r-A-\eta), r-A-\eta])-Q([-(r-A-\eta), r-A-\eta]) \\
& \quad=P(I(r-A-\eta))+Q(I(r-A-\eta)),
\end{aligned}
$$

because it is shown in [7] that any rectangle is a continuity set with respect to $W$. Substituting (4.5) and the same estimate for $Q(I(r-A-\eta))$ to the right-hand side of the above, we obtain the upper-bound part of the following theorem, which is the main general result in the present paper.

TheOREM. Let $\varphi(s)$ be the Euler product defined by (2.1), satisfying the conditions stated in Section 2. Let $B_{n}$ be as in (3.2), A the constant defined in Section 3, and for any positive $r$ greater than $A$, we define $K$ by the condition (4.4). Then

$$
W(\mathbf{C}-R(r), \sigma ; \varphi) \leq 4 \exp \left\{-(3 / 4)\left(\sum_{k=1}^{K}\left|B_{k}\right|\right)^{2}\left(\sum_{k>K}\left|B_{k}\right|^{2}\right)^{-1}\right\} .
$$

Moreover, let

$$
\left|B_{k(1)}\right| \geq\left|B_{k(2)}\right| \geq \ldots \geq\left|B_{k(l)}\right| \geq \ldots
$$

be a decreasing rearrangement of $\left\{\left|B_{k}\right|\right\}_{k=1}^{\infty}$ (which tends to 0 as $k \rightarrow \infty$ ). If we define $L=L(r)$ by the condition

$$
2^{-1} \sum_{l=1}^{L-1}\left|B_{k(l)}\right| \leq r+A<2^{-1} \sum_{l=1}^{L}\left|B_{k(l)}\right|
$$

then

$$
W(\mathbf{C}-R(r), \sigma ; \varphi) \geq 2^{-39} \exp \left\{-100\left(\sum_{l=1}^{L}\left|B_{k(l)}\right|\right)^{2}\left(\sum_{l>L}\left|B_{k(l)}\right|^{2}\right)^{-1}\right\} .
$$

Before starting the proof of the lower-bound part of this theorem, we note that if $\sum_{k=1}^{\infty}\left|B_{k}\right|<\infty$, then for sufficiently large $r$, it is impossible to choose $K$ (resp. $L$ ) satisfying (4.4) (resp. (4.7)), hence the theorem has no meaning. In this case, the Euler product is convergent absolutely, so trivially we have $W(\mathbf{C}-R(r), \sigma ; \varphi)=0$ for sufficiently large $r$.

In case $\sum_{k=1}^{\infty}\left|B_{k}\right|=\infty$, the above theorem gives upper and lower bounds of $W(\mathbf{C}-R(r), \sigma ; \varphi)$, but the results include sums of $\left|B_{k}\right|$ or $\left|B_{k(l)}\right|$. The behaviour of these sums depends on arithmetic properties of $\varphi(s)$, so it is, in general, not so easy to represent these sums in an explicit form. In the case of the Riemann zeta-function, Joyner has succeeded in obtaining the 
explicit form (1.2), by means of the prime number theorem. In the next two sections, we discuss how we can deduce the explicit estimates (Corollaries 1 and 2) from the above theorem, in the case of Dedekind zeta-functions.

Now we prove the lower-bound part of the theorem. By the second assertion of Lemma 2, we have

$$
\begin{aligned}
\mu_{\infty}\left(X^{*}(\bar{u})>2^{-1}\right. & \left.\sum_{l=1}^{L}\left|B_{k(l)}\right|\right) \\
& \geq 2^{-40} \exp \left\{-100\left(\sum_{l=1}^{L}\left|B_{k(l)}\right|\right)^{2}\left(\sum_{l>L}\left|B_{k(l)}\right|^{2}\right)^{-1}\right\},
\end{aligned}
$$

where

$$
X^{*}(\bar{u})=\sum_{l=1}^{\infty}\left|B_{k(l)}\right| \cos \left(2 \pi u_{l}\right) .
$$

We define the probability measure $P^{*}$ by $P^{*}(E)=\mu_{\infty}\left(X^{*-1}(E)\right)$ for any Borel set $E \subset \mathbf{R}$, and put

$$
\eta=2^{-1} \sum_{l=1}^{L}\left|B_{k(l)}\right|-(r+A)
$$

Then (4.9) implies

$$
P^{*}(I(r+A+\eta)) \geq 2^{-39} \exp \left\{-100\left(\sum_{l=1}^{L}\left|B_{k(l)}\right|\right)^{2}\left(\sum_{l>L}\left|B_{k(l)}\right|^{2}\right)^{-1}\right\}
$$

On the other hand, from (3.4) we can deduce

$$
W(\mathbf{C}-R(r), \sigma ; \varphi) \geq P(I(r+A+\eta)),
$$

by the method quite similar to that in the proof of the upper-bound part. Hence, the desired result follows immediately from (4.10) and (4.11), if we can show

$$
P^{*}(I(r+A+\eta))=P(I(r+A+\eta)) .
$$

Since the series defining $X$ and $X^{*}$ are not absolutely convergent, the validity of (4.12) is not a trivial fact. The following proof of (4.12) has been obtained in a discussion with Professor Tetsuya Hattori.

Lemma 3. Let $\psi\left(\right.$ resp. $\left.\psi^{*}\right)$ be the characteristic function of the probability measure $P\left(\right.$ resp. $\left.P^{*}\right)$. Then $\psi=\psi^{*}$.

Proof. For any real number $t$,

$$
\psi(t)=\int_{-\infty}^{\infty} \exp (i t x) d P(x)=\int_{\mathbb{T}^{\infty}} \exp (i t X(\bar{u})) d \mu_{\infty}(\bar{u})
$$




$$
=\prod_{k=1}^{\infty} \int_{0}^{1} \exp \left(i t\left|B_{k}\right| \cos \left(2 \pi u_{k}\right)\right) d u_{k}=\prod_{k=1}^{\infty} I\left(i t\left|B_{k}\right|\right)
$$

where

$$
I(r)=\int_{0}^{1} \exp (r \cos (2 \pi \theta)) d \theta .
$$

In Montgomery [9], the expansion

$$
I(r)=\sum_{n=0}^{\infty}(n !)^{-2}(r / 2)^{2 n}
$$

is proved. Hence we have

$$
I\left(i t\left|B_{k}\right|\right)=1+O\left(\left|t B_{k}\right|^{2}\right),
$$

which implies, with (4.2), the absolute convergence of the infinite product (4.13). Therefore, the order of the product (4.13) can be changed freely. In particular, the assertion $\psi=\psi^{*}$ follows.

Now we obtain (4.12) as a direct consequence of Lemma 3. This completes the proof of the lower-bound part of the theorem.

5. Proof of Corollary 2. In case $\varphi(s)=\zeta_{F}(s)$, from (3.2) we have

$$
\left|B_{k}\right|=p_{k}^{-\sigma} \cdot \#\{j \mid 1 \leq j \leq g(k), f(j, k)=1\} .
$$

In this section, we assume $F$ is a Galois extension of $\mathbf{Q}$. Let

$$
\mathfrak{K}=\{k \mid e(k)>1, f(k)=1\} .
$$

Then $\mathfrak{K}$ is a finite set, and for any $k$ which does not belong to $\mathfrak{K}$,

$$
\left|B_{k}\right|= \begin{cases}d \cdot p_{k}^{-\sigma} & \text { if } g(k)=d, \\ 0 & \text { otherwise. }\end{cases}
$$

We choose $r_{0}$ so large that $k<K\left(r_{0}\right)$ for any $k \in \mathfrak{K}$. Then for any $r \geq r_{0}$, we have

$$
\sum_{k=1}^{K}\left|B_{k}\right|=d \sum_{\substack{1 \leq k \leq K \\ g(k)=d}} p_{k}^{-\sigma}+O(1)
$$

where the term $O(1)$ comes from the finitely many $k$ 's belonging to $\mathfrak{K}$, and

$$
\sum_{k>K}\left|B_{k}\right|^{2}=d^{2} \sum_{\substack{k>K \\ g(k)=d}} p_{k}^{-2 \sigma} .
$$

For any positive $w$, we put

$$
\varrho(w)=\#\left\{p_{k} \leq w \mid g(k)=d\right\} .
$$


Then it is a direct consequence of the prime ideal theorem that

$$
\varrho(w)=d^{-1} \frac{w}{\log w}(1+o(1)) .
$$

By using this asymptotic formula, we calculate the right-hand sides of (5.2) and (5.3). In the following calculations we sometimes change the integration symbol and the factor $(1+o(1))$, the validity of this procedure we can easily check.

Let $x$ be a real number satisfying $p_{K} \leq x<p_{K+1}$. Then, by partial summation, from (5.3) and (5.4) it follows that

$$
\begin{aligned}
& \sum_{k>K}\left|B_{k}\right|^{2}=2 \sigma d^{2} \int_{x}^{\infty}(\varrho(\xi)-\varrho(x)) \xi^{-2 \sigma-1} d \xi \\
& =2 \sigma d(1+o(1)) \int_{x}^{\infty} \frac{\xi^{-2 \sigma}}{\log \xi} d \xi-2 \sigma d \frac{x}{\log x}(1+o(1)) \int_{x}^{\infty} \xi^{-2 \sigma-1} d \xi \\
& =\frac{d}{2 \sigma-1} \frac{x^{1-2 \sigma}}{\log x}(1+o(1)),
\end{aligned}
$$

because integration by parts gives

$$
\int_{x}^{\infty} \frac{\xi^{-2 \sigma}}{\log \xi} d \xi=\frac{1}{2 \sigma-1} \frac{x^{1-2 \sigma}}{\log x}(1+o(1)) .
$$

Next, in case $1-d_{1}^{-1}<\sigma<1$, from (5.2) and (5.4) we have

$$
\begin{aligned}
\sum_{k=1}^{K}\left|B_{k}\right| & =d \varrho(x) x^{-\sigma}+d \sigma \int_{2}^{x} \varrho(\xi) \xi^{-\sigma-1} d \xi+O(1) \\
& =\frac{x^{1-\sigma}}{\log x}(1+o(1))+\sigma(1+o(1)) \int_{2}^{x} \frac{\xi^{-\sigma}}{\log \xi} d \xi \\
& =\frac{1}{1-\sigma} \frac{x^{1-\sigma}}{\log x}(1+o(1)),
\end{aligned}
$$

because

$$
\int_{2}^{x} \frac{\xi^{-\sigma}}{\log \xi} d \xi=\frac{1}{1-\sigma} \frac{x^{1-\sigma}}{\log x}(1+o(1)) .
$$

In case $\sigma=1$, we have

$$
\sum_{k=1}^{K}\left|B_{k}\right|=d \varrho(x) x^{-1}+d \int_{2}^{x} \varrho(\xi) \xi^{-2} d \xi+O(1)
$$




$$
=(1+o(1)) \int_{2}^{x} \frac{d \xi}{\xi \log \xi}+O(1)=(\log \log x)(1+o(1)) .
$$

Therefore, by the upper-bound part (4.6) of our theorem, we obtain

$$
W\left(\mathbf{C}-R(r), \sigma ; \zeta_{F}\right) \leq\left\{\begin{array}{c}
4 \exp \left\{-\frac{3(2 \sigma-1)}{4(1-\sigma)^{2}} d^{-1} \frac{x}{\log x}(1+o(1))\right\} \\
\text { if } 1-d_{1}^{-1}<\sigma<1, \\
4 \exp \left\{-(3 / 4) d^{-1} x(\log x)(\log \log x)^{2}(1+o(1))\right\} \\
\text { if } \sigma=1 .
\end{array}\right.
$$

From (4.4), (5.6) and (5.7), we have

$$
r= \begin{cases}\frac{2}{1-\sigma} \frac{x^{1-\sigma}}{\log x}(1+o(1)) & \text { if } 1-d_{1}^{-1}<\sigma<1, \\ 2(\log \log x)(1+o(1)) & \text { if } \sigma=1 .\end{cases}
$$

Hence, in case $\sigma=1$, we have

$$
x=\exp \exp \left(2^{-1} r(1+o(1))\right) .
$$

In case $1-d_{1}^{-1}<\sigma<1$, let

$$
r_{1}=\frac{2}{1-\sigma} \frac{x^{1-\sigma}}{\log x} .
$$

Then $r_{1}=r(1+o(1))$, and

SO

$$
r_{1}^{1 /(1-\sigma)}\left(\log r_{1}\right)^{\sigma /(1-\sigma)}=2^{1 /(1-\sigma)}(1-\sigma)^{-1} \frac{x}{\log x}(1+o(1)),
$$

$$
\frac{x}{\log x}=(1-\sigma) 2^{-1 /(1-\sigma)} r^{1 /(1-\sigma)}(\log r)^{\sigma /(1-\sigma)}(1+o(1)) .
$$

Substituting (5.9) and (5.10) in (5.8), we obtain the upper-bound part of Corollary 2.

Next we prove the lower-bound estimates. We define the value of $L$ by the condition (4.7). Let $y$ be a real number satisfying $p_{k(L)} \leq y<p_{k(L)+1}$. Since the subsequence $\left\{\left|B_{k}\right| \mid g(k)=d\right\}$ is decreasing, we can assume, for sufficiently large $r_{0}$, that $\left|B_{k}\right| \geq\left|B_{k(L)}\right|$ for any $k \in \mathfrak{K}$ and any $r \geq r_{0}$. Then

$$
\sum_{l=1}^{L}\left|B_{k(l)}\right|=d \sum_{\substack{p<y \\ g(p)=d}} p^{-\sigma}+O(1)
$$

and

$$
\sum_{l>L}\left|B_{k(l)}\right|^{2}=d^{2} \sum_{\substack{p>y \\ g(p)=d}} p^{-2 \sigma}
$$


Calculating the right-hand sides of these equations by the same method as above, we can deduce the lower-bound part of Corollary 2, by using (4.8) instead of (4.6).

6. Proof of Corollary 1. In this section we assume $F$ is an arbitrary finite extension of Q. If $g^{*}(k)=d^{*}$, then it is obvious that $g(k)=d$, so $\left|B_{k}\right|=d p_{k}^{-\sigma}$. Hence, for any $x \in\left[p_{K}, p_{K+1}\right)$, we have

$$
\sum_{k=1}^{K}\left|B_{k}\right| \geq d \sum_{\substack{p \leq x \\ g^{*}(p)=d^{*}}} p^{-\sigma}
$$

We put

$$
\varrho^{*}(w)=\#\left\{p_{n} \leq w \mid g^{*}(n)=d^{*}\right\}
$$

for any $w>0$. Then by the prime ideal theorem we have

$$
\varrho^{*}(w)=\left(d^{*}\right)^{-1} \frac{w}{\log w}(1+o(1)) .
$$

By using this formula, we can calculate the right-hand side of (6.1) as in the previous section, to obtain

$$
\sum_{k=1}^{K}\left|B_{k}\right| \geq \begin{cases}\left(d / d^{*}\right)(1-\sigma)^{-1} \frac{x^{1-\sigma}}{\log x}(1+o(1)) & \text { if } 1-d_{1}^{-1}<\sigma<1 \\ \left(d / d^{*}\right)(\log \log x)(1+o(1)) & \text { if } \sigma=1\end{cases}
$$

Next, it is clear that

$$
\sum_{k>K}\left|B_{k}\right|^{2} \leq d^{2} \sum_{p>x} p^{-2 \sigma},
$$

and, calculating the right-hand side of the above by using the prime number theorem and partial summation, we have

$$
\sum_{k>K}\left|B_{k}\right|^{2} \leq d^{2}(2 \sigma-1)^{-1} \frac{x^{1-2 \sigma}}{\log x}(1+o(1)) .
$$

Also, since

$$
\sum_{k=1}^{K+1}\left|B_{k}\right| \leq d \sum_{k \leq K+1} p_{k}^{-\sigma}
$$

with (4.4) we have

$$
r \leq \begin{cases}2 d(1-\sigma)^{-1} \frac{x^{1-\sigma}}{\log x}(1+o(1)) & \text { if } 1-d_{1}^{-1}<\sigma<1, \\ 2 d(\log \log x)(1+o(1)) & \text { if } \sigma=1,\end{cases}
$$

by using the prime number theorem. Hence

$$
x \geq \exp \exp \left((2 d)^{-1} r(1+o(1))\right)
$$


if $\sigma=1$, and

$$
\frac{x}{\log x} \geq(1-\sigma) 2^{-1 /(1-\sigma)} d^{-1 /(1-\sigma)} r^{1 /(1-\sigma)}(\log r)^{\sigma /(1-\sigma)}(1+o(1))
$$

if $1-d_{1}^{-1}<\sigma<1$. On the other hand, substituting (6.3) and (6.4) in (4.6), we have

$$
W\left(\mathbf{C}-R(r), \sigma ; \zeta_{F}\right) \leq\left\{\begin{array}{c}
4 \exp \left\{-\frac{3(2 \sigma-1)}{4(1-\sigma)^{2}}\left(d^{*}\right)^{-2} \frac{x}{\log x}(1+o(1))\right\} \\
\text { if } 1-d_{1}^{-1}<\sigma<1, \\
4 \exp \left\{-(3 / 4)\left(d^{*}\right)^{-2} x(\log x)(\log \log x)^{2}(1+o(1))\right\} \\
\text { if } \sigma=1 .
\end{array}\right.
$$

Therefore, using (6.6) and (6.7), we obtain the upper-bound estimates in Corollary 1.

In order to deduce the lower-bound part of Corollary 1, we first prove that

$$
\sum_{l \leq L}\left|B_{k(l)}\right| \leq \begin{cases}(1-\sigma) d \frac{z^{1-\sigma}}{\log z}(1+o(1)) & \text { if } 1-d_{1}^{-1}<\sigma<1 \\ d(\log \log z)(1+o(1)) & \text { if } \sigma=1\end{cases}
$$

where $z$ is a real number satisfying $p_{L} \leq z<p_{L+1}$. In fact, $\left|B_{k(l)}\right| \leq d p_{l}^{-\sigma}$ for any $l$, because $\left|B_{n}\right| \leq d p_{n}^{-\sigma} \leq d p_{l}^{-\sigma}$ for any $n \geq l$. Hence,

$$
\sum_{l \leq L}\left|B_{k(l)}\right| \leq d \sum_{l \leq L} p_{l}^{-\sigma}
$$

To obtain (6.8), it is enough to evaluate the right-hand side of the above by using the prime number theorem, as in the proof of (6.5).

Next, let $\left\{p_{j(l)}\right\}_{l=1}^{\infty}$ be the increasing sequence of all prime numbers which satisfy $g^{*}\left(p_{j(l)}\right)=d^{*}$. Then

$$
\left|B_{k(l)}\right| \geq\left|B_{j(l)}\right|=d p_{j(l)}^{-\sigma},
$$

so, by using (6.2), we have

$$
\sum_{l>L}\left|B_{k(l)}\right|^{2} \geq d^{2} \sum_{\substack{p>y \\ g^{*}(p)=d^{*}}} p^{-2 \sigma}=(2 \sigma-1)^{-1} d^{2}\left(d^{*}\right)^{-1} \frac{y^{1-2 \sigma}}{\log y}(1+o(1))
$$

where $y \in\left[p_{j(L)}, p_{j(L)+1}\right)$. Since

$$
L=\varrho^{*}\left(p_{j(L)}\right)=\left(d^{*}\right)^{-1} \frac{p_{j(L)}}{\log p_{j(L)}}(1+o(1))
$$

we have

$$
p_{j(L)}=d^{*} L(\log L)(1+o(1)) .
$$


Hence, comparing with $z=L(\log L)(1+o(1))$, we have $y=d^{*} z(1+o(1))$, and (6.9) implies

$$
\sum_{l>L}\left|B_{k(l)}\right|^{2} \geq(2 \sigma-1)^{-1} d^{2}\left(d^{*}\right)^{-2 \sigma} \frac{z^{1-2 \sigma}}{\log z}(1+o(1)) .
$$

From (4.8), (6.8) and the above, we have

$$
W\left(\mathbf{C}-R(r), \sigma ; \zeta_{F}\right) \geq\left\{\begin{array}{c}
2^{-39} \exp \left\{-100 \frac{2 \sigma-1}{(1-\sigma)^{2}}\left(d^{*}\right)^{2 \sigma} \frac{z}{\log z}(1+o(1))\right\} \\
\text { if } 1-d_{1}^{-1}<\sigma<1, \\
2^{-39} \exp \left\{-100\left(d^{*}\right)^{2} z(\log z)(\log \log z)^{2}(1+o(1))\right\} \\
\text { if } \sigma=1 .
\end{array}\right.
$$

Lastly, since $L$ was chosen to satisfy (4.7), we have

$$
r+A \geq 2^{-1} \sum_{l=1}^{L-1}\left|B_{k(l)}\right| \geq 2^{-1} \sum_{l=1}^{L-1}\left|B_{l}\right| .
$$

Hence, combining with (6.3), we have

$$
r \geq \begin{cases}(1-\sigma)^{-1}\left(d / 2 d^{*}\right) \frac{z^{1-\sigma}}{\log z}(1+o(1)) & \text { if } 1-d_{1}^{-1}<\sigma<1, \\ \left(d / 2 d^{*}\right)(\log \log z)(1+o(1)) & \text { if } \sigma=1 .\end{cases}
$$

We can deduce the lower-bounds in Corollary 1 from these inequalities, similarly to the upper-bound case.

7. Other examples. In this section, we discuss two further examples. Let $q \in \mathbf{N}, \chi$ a Dirichlet character $\bmod q$, and

$$
L(s, \chi)=\prod_{k=1}^{\infty}\left(1-\chi\left(p_{k}\right) p_{k}^{-s}\right)^{-1}
$$

the corresponding Dirichlet $L$-function. Then $\left|B_{k}\right|=p_{k}^{-\sigma}\left|\chi\left(p_{k}\right)\right|$ is decreasing except for finitely many $k$ 's for which $p_{k}$ 's are prime divisors of $q$. Hence, by the same method as described in Section 5 , we obtain

$$
\begin{aligned}
2^{-39} & \exp \left\{-100 \frac{2 \sigma-1}{1-\sigma} 2^{1 /(1-\sigma)} r^{1 /(1-\sigma)}(\log r)^{\sigma /(1-\sigma)}(1+o(1))\right\} \\
& \leq W(\mathbf{C}-R(r), \sigma ; L) \\
& \leq 4 \exp \left\{-(3 / 4) \frac{2 \sigma-1}{1-\sigma} 2^{-1 /(1-\sigma)} r^{1 /(1-\sigma)}(\log r)^{\sigma /(1-\sigma)}(1+o(1))\right\}
\end{aligned}
$$

for $1 / 2<\sigma<1$, and

$$
\begin{aligned}
2^{-39} \exp \{-100 \exp \exp (2 r(1 & +o(1)))\} \leq W(\mathbf{C}-R(r), 1 ; L) \\
& \leq 4 \exp \left\{-(3 / 4) \exp \exp \left(2^{-1} r(1+o(1))\right)\right\} .
\end{aligned}
$$


In case $1 / 2<\sigma<1$, the above has already been obtained in Joyner [5], though the coefficients are not explicit in his statement.

Next, let $f$ be a primitive form of weight $m$ with respect to the full modular group $\mathrm{SL}(2, \mathbf{Z})$. The corresponding Dirichlet series $\varphi_{f}(s)$ can be written as the following Euler product:

$$
\varphi_{f}(s)=\prod_{k=1}^{\infty}\left\{\left(1-\alpha_{k} p_{k}^{-s}\right)\left(1-\beta_{k} p_{k}^{-s}\right)\right\}^{-1},
$$

and $c\left(p_{k}\right)=\alpha_{k}+\beta_{k}$ is the $p_{k}$ th Fourier coefficient of $f$. The existence of the asymptotic probability measure $W\left(R, \sigma ; \varphi_{f}\right)$ for any $\sigma>m / 2$ has already been proved in [6].

Let $\lambda \in[0,1] \cup[2, \infty), \alpha(p)=c(p) p^{(1-m) / 2}$, and

$$
P(x, 2 \lambda)=\sum_{p \leq x}|\alpha(p)|^{2 \lambda} .
$$

Then Rankin [13] proved

$$
\begin{aligned}
2^{\lambda-1} & \leq \liminf _{x \rightarrow \infty} x^{-1} P(x, 2 \lambda) \log x \\
& \leq \limsup _{x \rightarrow \infty} x^{-1} P(x, 2 \lambda) \log x \leq \frac{2^{\lambda-1}}{5}\left(2^{\lambda}+3^{2-\lambda}\right) .
\end{aligned}
$$

In particular,

$$
\sum_{p \leq x}|\alpha(p)|^{2}=\frac{x}{\log x}(1+o(1))
$$

and

$$
\frac{1}{\sqrt{2}} \frac{x}{\log x}(1+o(1)) \leq \sum_{p \leq x}|\alpha(p)| \leq \frac{2+3 \sqrt{6}}{10} \frac{x}{\log x}(1+o(1)) .
$$

By using these inequalities, we can show the following upper-bound estimates of $W\left(\mathbf{C}-R(r), \sigma ; \varphi_{f}\right)$, in a way similar to that in Section 5 .

COROLlaRY 3. There exists a positive constant $M_{3}$, which depends only on $m$ and $\sigma$, such that

$$
\begin{aligned}
W(\mathbf{C}- & \left.R(r), \sigma ; \varphi_{f}\right) \\
& \leq 4 \exp \left\{-M_{3} r^{2 /(m+1-2 \sigma)}(\log r)^{(2 \sigma-m+1) /(m+1-2 \sigma)}(1+o(1))\right\}
\end{aligned}
$$

for $m / 2<\sigma<(m+1) / 2$. Also,

$$
\begin{aligned}
W\left(\mathbf{C}-R(r),(m+1) / 2 ; \varphi_{f}\right) & \\
& \leq 4 \exp \left\{-(3 / 8) \exp \exp \left(5(2+3 \sqrt{6})^{-1} r(1+o(1))\right)\right\} .
\end{aligned}
$$


R e mark. A possible choice of $M_{3}$ is

$$
M_{3}=\frac{3(2 \sigma-m)}{4(m+1-2 \sigma)}\left(\frac{2+3 \sqrt{6}}{5}\right)^{-2 /(m+1-2 \sigma)} .
$$

In general, if we have arithmetic information of the types (7.1) and (7.2) on the Euler product $\varphi(s)$ defined by $(2.1)$, then we can deduce an explicit upper-bound estimate of $W(\mathbf{C}-R(r), \sigma ; \varphi)$, which will improve (2.5). However, to obtain an explicit lower-bound estimate is, generally, a more difficult problem. Recently, a lower-bound estimate of $W\left(\mathbf{C}-R(r), \sigma ; \varphi_{f}\right)\left(\varphi_{f}\right.$ is as in Corollary 3) has been obtained by using a result of M. Ram Murty. This work will be published in [3].

8. A Jessen-Wintner type inequality. In this section, we give a proof of the inequality (2.5). If we can evaluate the right-hand side of (4.6) explicitly, as in the case of Corollaries 1 to 3, it certainly gives a better estimate than (2.5). However, as we have seen in the previous sections, such an evaluation requires a "prime-number-theorem type" result, which has not been known for general Euler products. Therefore, as a general explicit estimate, (2.5) remains the best.

In case $\varphi(s)$ is the Dirichlet series attached to a primitive form with respect to some Hecke congruence subgroup of $\mathrm{SL}(2, \mathbf{Z})$, the inequality $(2.5)$ has already been proved in [6]. The following proof is a simple generalization of the one in [6].

Let $\sigma>\varrho$, and let $\lambda$ be an arbitrary real number. We claim that there exists a positive $a_{0}=a_{0}(\lambda, \sigma ; \varphi)$ such that

$$
W_{N}(E, \sigma ; \varphi) \leq C \exp \left(-\lambda a^{2}\right)
$$

for any $a>a_{0}$, any Borel set $E \subset\{z|| z \mid>2 a\}$ and any sufficiently large positive integer $N$, with a positive constant $C=C(\lambda, \sigma ; \varphi)$. We can easily deduce (2.5) from (8.1), by a standard argument (see Section 5 of $[6]$ ).

Let $r$ be a positive integer, $N>r$, and put

$$
S_{r, N}\left(\theta_{r+1}, \ldots, \theta_{N}\right)=-\sum_{n=r+1}^{N} \sum_{j=1}^{g(n)} \log \left(1-a_{n}^{(j)} p_{n}^{-f(j, n) \sigma} \exp \left(2 \pi i f(j, n) \theta_{n}\right)\right) .
$$

We define the probability measure $W_{r, N}$ by $W_{r, N}(E)=\mu_{N-r}\left(S_{r, N}^{-1}(E)\right)$ for any Borel set $E$. Then, by the same method as that described in the proof of Lemma 2 in [6], we can prove (8.1) by using the following

Lemma 4. Let $\lambda>0, b>0$, and $B$ a bounded set which satisfies $B \subset\{z \mid$ $|z| \leq b\}$. Then there exists a sufficiently large positive integer $r=r(\lambda, \sigma ; \varphi)$ such that

$$
W_{r, N}\left(z_{0}-B\right) \leq C_{1} \exp \left(-4 \lambda\left|z_{0}\right|^{2}\right)
$$


for any $N>r$ and any $z_{0} \in\{z|| z \mid>2 b\}$, with a positive constant $C_{1}=$ $C_{1}(\lambda, \sigma ; \varphi)$. (Here $z_{0}-B$ is the set $\left\{z_{0}-w \mid w \in B\right\}$.)

Proof. If $\theta_{r, N}=\left(\theta_{r+1}, \ldots, \theta_{N}\right) \in S_{r, N}^{-1}\left(z_{0}-B\right)$, then $\left|S_{r, N}\left(\theta_{r, N}\right)\right|>$ $\left|z_{0}\right| / 2$. Hence

(8.2) $\exp \left(4 \lambda\left|z_{0}\right|^{2}\right) W_{r, N}\left(z_{0}-B\right)$

$$
\begin{aligned}
& =\int_{S_{r, N}^{-1}\left(z_{0}-B\right)} \exp \left(4 \lambda\left|z_{0}\right|^{2}\right) d \mu_{N-r}\left(\theta_{r, N}\right) \\
& \leq \int_{S_{r, N}^{-1}\left(z_{0}-B\right)} \exp \left(16 \lambda\left|S_{r, N}\left(\theta_{r, N}\right)\right|^{2}\right) d \mu_{N-r}\left(\theta_{r, N}\right) \\
& \leq \int_{\mathbb{T}^{N-r}} \exp \left(16 \lambda\left|S_{r, N}\left(\theta_{r, N}\right)\right|^{2}\right) d \mu_{N-r}\left(\theta_{r, N}\right) .
\end{aligned}
$$

We put

$$
S_{r, N}^{*}\left(\theta_{r, N}\right)=\sum_{n=r+1}^{N} B_{n} \exp \left(2 \pi i \theta_{n}\right) .
$$

Then, similarly to Section 3, we obtain

$$
\left|S_{r, N}\left(\theta_{r, N}\right)-S_{r, N}^{*}\left(\theta_{r, N}\right)\right| \leq A .
$$

In general, if $|u-v| \leq w$, then $|u|^{2} \leq 2\left(|v|^{2}+w^{2}\right)$. Hence, from (8.3) we have

$$
\begin{aligned}
\int_{\mathbb{T}^{N-r}} & \exp \left(16 \lambda\left|S_{r, N}\left(\theta_{r, N}\right)\right|^{2}\right) d \mu_{N-r}\left(\theta_{r, N}\right) \\
& \leq \int_{\mathbb{T}^{N-r}} \exp \left(32 \lambda\left(\left|S_{r, N}^{*}\left(\theta_{r, N}\right)\right|^{2}+A^{2}\right)\right) d \mu_{N-r}\left(\theta_{r, N}\right) \\
& =\exp \left(32 \lambda A^{2}\right) \sum_{k=0}^{\infty} \frac{(32 \lambda)^{k}}{k !} \int_{\mathbb{T}^{N-r}}\left|S_{r, N}^{*}\left(\theta_{r, N}\right)\right|^{2 k} d \mu_{N-r}\left(\theta_{r, N}\right) .
\end{aligned}
$$

Now, we have

$$
\begin{aligned}
\int_{\mathbb{T}^{N-r}}\left|S_{r, N}^{*}\left(\theta_{r, N}\right)\right|^{2 k} d \mu_{N-r}\left(\theta_{r, N}\right) \\
=\sum_{\substack{k_{r+1}+\ldots+k_{N}=k \\
j_{r+1}+\ldots+j_{N}=k}} \frac{(k !)^{2}}{k_{r+1} ! \ldots k_{N} ! j_{r+1} ! \ldots j_{N} !} \\
\quad \times \prod_{n=r+1}^{N} B_{n}^{k_{n}} \bar{B}_{n}^{j_{n}} \int_{0}^{1} \exp \left(2 \pi i\left(k_{n}-j_{n}\right) \theta_{n}\right) d \theta_{n}
\end{aligned}
$$




$$
=\sum_{k_{r+1}+\ldots+k_{N}=k}\left(\frac{k !}{k_{r+1} ! \ldots k_{N} !}\right)^{2} \prod_{n=r+1}^{N}\left|B_{n}\right|^{2 k_{n}} \leq k !\left(\sum_{n=r+1}^{N}\left|B_{n}\right|^{2}\right)^{k}
$$

so, together with (8.2) and (8.4), we obtain

$$
\exp \left(4 \lambda\left|z_{0}\right|^{2}\right) W_{r, N}\left(z_{0}-B\right) \leq \exp \left(32 \lambda A^{2}\right) \sum_{k=0}^{\infty}\left(32 \lambda \sum_{n=r+1}^{N}\left|B_{n}\right|^{2}\right)^{k}
$$

It follows from (4.2) that for sufficiently large $r=r(\lambda, \sigma ; \varphi)$, we have

$$
d=1-32 \lambda \sum_{n=r+1}^{\infty}\left|B_{n}\right|^{2} \geq 1 / 2 \text {. }
$$

Hence, for any $N>r$,

$$
\sum_{k=0}^{\infty}\left(32 \lambda \sum_{n=r+1}^{N}\left|B_{n}\right|^{2}\right)^{k} \leq \sum_{k=0}^{\infty}(1-d)^{k} \leq \sum_{k=0}^{\infty} 2^{-k}=2 .
$$

Therefore, the assertion of Lemma 4 follows from (8.5). The validity of the inequality (2.5) is now established.

\section{References}

[1] H. Bohr und B. Jessen, Über die Wertverteilung der Riemannschen Zetafunktion, Erste Mitteilung, Acta Math. 54 (1930), 1-35; Zweite Mitteilung, ibid. 58 (1932), $1-55$.

[2] K. Chandrasekharan and R. Narasimhan, The approximate functional equation for a class of zeta-functions, Math. Ann. 152 (1963), 30-64.

[3] T. Hattori and K. Matsumoto, Large deviations of Montgomery type and its application to the theory of zeta-functions, preprint.

[4] B. Jessen and A. Wintner, Distribution functions and the Riemann zeta function, Trans. Amer. Math. Soc. 38 (1935), 48-88.

[5] D. Joyner, Distribution Theorems of L-functions, Longman Scientific \& Technical, 1986.

[6] K. Matsumoto, A probabilistic study on the value-distribution of Dirichlet series attached to certain cusp forms, Nagoya Math. J. 116 (1989), 123-138.

[7] —, Value-distribution of zeta-functions, in: Analytic Number Theory, Proceedings of the Japanese-French Symposium held in Tokyo, Oct. 10-13, 1988, K. Nagasaka and E. Fouvry (eds.), Lecture Notes in Math. 1434, Springer, 1990, 178-187.

[8] —, Asymptotic probability measures of zeta-functions of algebraic number fields, J. Number Theory, to appear.

[9] H. L. Montgomery, The zeta function and prime numbers, in: Proceedings of the Queen's Number Theory Conference, 1979, P. Ribenboim (ed.), Queen's Papers in Pure and Appl. Math. 54, Queen's Univ., Kingston, Ont., 1980, 1-31.

[10] H. L. Montgomery and A. M. Odlyzko, Large deviations of sums of independent random variables, Acta Arith. 49 (1988), 427-434. 
[11] E. M. Nikishin, Dirichlet series with independent exponents and some of their applications, Mat. Sb. 96 (138) (1975), 3-40 = Math. USSR-Sb. 25 (1975), 1-36.

[12] H. S. A. Potter, The mean values of certain Dirichlet series I, Proc. London Math. Soc. 46 (1940), 467-478.

[13] R. A. Rankin, Sums of powers of cusp form coefficients II, Math. Ann. 272 (1985), 593-600.

DEPARTMENT OF MATHEMATICS

FACULTY OF EDUCATION

IWATE UNIVERSITY

UEDA, MORIOKA 020, JAPAN 